\title{
More Optimal Packings of Equal Circles in a Square*
}

\author{
K. J. Nurmela and P. R. J. Östergård \\ Department of Computer Science and Engineering, \\ Helsinki University of Technology, \\ P.O. Box 5400, FIN-02015 HUT, Finland \\ \{Kari.Nurmela,Patric.Ostergard\}@ hut.fi
}

\begin{abstract}
The densest packings of $n$ equal circles in a square have been determined earlier for $n \leq 20$ and for $n=25,36$. Several of these packings have been proved with the aid of a computer. The computer-aided approach is further developed here and the range is extended to $n \leq 27$. The optimal packings are depicted.
\end{abstract}

\section{Introduction}

We consider the following packing problem: Find the value of $r_{n}$, the maximum radius of $n$ nonoverlapping equal circles in a unit square. Another similar problem is that of finding $d_{n}$, the maximum possible minimum distance between $n$ points in a unit square. Actually, it is not difficult to show that these two problems are equivalent and that $r_{n}=d_{n} /\left(2 d_{n}+2\right)$. A packing of circles with maximum radius (and the corresponding spread of points) is called optimal.

Solved and unsolved packing problems have been thoroughly discussed in [1], [4], and [13]. Circle packings in a square have been considered in the mathematical literature since the early 1960s when the problem was raised by Moser [15]. During the last three decades, optimality proofs have been published for the following values of $n$ : $1-9$ (in [12], [21], [23], and [25]), 14 (in [30]), 16 (in [28]), 25 (in [29]), and 36 (in [10]). Constructions of good packings for other values of $n$ have been published in, for example, [5], [7], [14], [16], [22], [24], and [27].

Recently, optimal packings for $n \leq 20$ were determined and the corresponding values of $d_{n}$ were obtained as explicit values or as the smallest positive root of a polynomial

\footnotetext{
* The research of K. J. Nurmela was supported by the Emil Aaltonen Foundation; the Vilho, Yrjö, and Kalle Väisälä Foundation; and the Academy of Finland. The research of P. R. J. Östergård was supported by the Academy of Finland.
} 
equation in one variable [2], [3], [19]. The proofs involve extensive use of computers and cannot be checked by hand. In this paper we verify these old results, enhance the approach, and extend the number of circles to 27. As usual in computer-aided proofs, the optimality proofs of packings in this paper consist of very long chains of automated, relatively simple steps. It is not possible (or reasonable) to list all these steps in this paper. Instead, we describe the algorithms used with such detail that it should be possible to reimplement our programs from scratch and verify the computational results. To facilitate possible re-implementation and further development of the algorithms, the most crucial data of our computer runs is included in this paper. We would further like to point out that it is not reasonable to try to automatize the whole proof in one computer program, but human interaction at different stages should be used; this speeds up the proof and makes it easier to follow the process. Preliminary results of this research have been published by the authors in [17].

Even if we here prove optimality only for packings of at most 27 circles, for larger $n$ we can still try to find as good packings as possible. In [16] packings of up to 50 circles are given, some of which are improved in [7]. All of the optimal packings for $n \leq 27$ are among those published in [7] and [16].

In Section 2 the computer-aided method used to prove optimality of packings is presented. Some statistics of the computer runs-both for the old $(n \leq 20)$ and the new cases (with some data in the Appendix) - are given in Section 3, which also contains pictures of the optimal packings for $21 \leq n \leq 27$. The paper is concluded in Section 4 .

\section{Computer-Aided Proof Method}

We here give a comprehensive description of the computer-aided proof procedure used. The proof procedure is based on that developed by de Groot et al. [2], [19]. All steps of that method have been modified, some only slightly and some more substantially.

\subsection{Overview of the Method}

We consider the problem of spreading $n$ points in a unit square. Initially, we tile the square in such a way that the maximum distance between two points in a single tile (the diameter of the tile) is smaller than the minimum distance between points in the best packing known. Consequently, no two points in an optimal packing can be in the same tile.

Next, we consider - up to symmetry - all possible ways of choosing $n$ of the tiles. For each of these combinations, the area of these tiles that can contain a point is reduced by considering the areas of other tiles. If this area of a tile completely vanishes in the process, the combination can be discarded, because it cannot contain an optimal packing. This leads to elimination of most of the combinations. Additional eliminations can be made by dividing a combination into subcases, which are eliminated one by one. An optimal combination is a combination that contains an optimal packing.

After the elimination process we know combinations of which at least one is optimal, and for each combination we further know small areas where the points of an optimal 
packing should reside. For a combination, we now take the best known packing with the points within the remaining areas, and prove that there are no better such packings. This is accomplished by a reduction process. The process-when successful-essentially shows that the areas where the points must reside in a packing at least as good as the prescribed packing can be made arbitrarily small and the conjectured packing is thus best possible and unique within the small areas that we started from. This leads to a finite number of packings out of which the optimal one(s) can be found.

In the following we describe each phase of the optimality proof algorithm in more detail.

\subsection{Finding Initial Combinations}

Initially, the square is tiled with congruent square or rectangular tiles in such a way that two points of an optimal packing cannot be in the same tile. This is achieved by selecting the size of tiles so that the diameter of the tiles is smaller than $d_{n}$, the distance between points in the optimal packing (point spread). Points lying on a tile boundary are considered as part of both tiles (or of all four tiles if the point is on a boundary crossing). Good lower bounds for $d_{n}$ when $n$ is not too large can be obtained by solving numerically with desired precision the distance between the points in the best known (often conjecturally optimal) packing.

Diameters of tiles and the cases the tilings can be applied to are shown in Table 1. The tilings that were used in the proofs will be given later for each case together with other statistics. In practice, it turns out that it is computationally advantageous for the next steps of the proof procedure to use square tiles (the larger the aspect ratio of the rectangles of a tiling is, the worse is the result of the elimination procedure to be described). If the square is tiled into rectangles, it is tiled so that there are fewer tiles in a row than in a column. (This is essential for the numbering of combinations, discussed below.)

We denote the total number of tiles by $t$ and consider all possible ways of choosing $n$ out of $t$ tiles. For a given choice, these $n$ tiles are assumed to contain a point and are

Table 1. Tilings of a square.

\begin{tabular}{|c|c|c|}
\hline Tiling & Diameter & $\begin{array}{c}\text { Maximum } \\
\text { number of } \\
\text { circles }\end{array}$ \\
\hline $2 \times 2$ & $\sqrt{2} / 2 \approx 0.707106781$ & 4 \\
\hline $2 \times 3$ & $\sqrt{13} / 6 \approx 0.600925213$ & 5 \\
\hline $3 \times 3$ & $\sqrt{2} / 3 \approx 0.471404521$ & 9 \\
\hline $3 \times 4$ & $5 / 12 \approx 0.416666667$ & 10 \\
\hline $4 \times 4$ & $\sqrt{2} / 4 \approx 0.353553391$ & 13 \\
\hline $4 \times 5$ & $\sqrt{41} / 20 \approx 0.320156212$ & 16 \\
\hline $4 \times 6$ & $\sqrt{13} / 12 \approx 0.300462606$ & 17 \\
\hline $5 \times 5$ & $\sqrt{2} / 5 \approx 0.282842712$ & 20 \\
\hline $5 \times 6$ & $\sqrt{61} / 30 \approx 0.260341656$ & 22 \\
\hline $6 \times 6$ & $\sqrt{2} / 6 \approx 0.235702260$ & 27 \\
\hline
\end{tabular}


called full; the rest of the tiles are called empty. By taking symmetries into account, we need not use all possible combinations in the computations: if a set of combinations can be obtained from each other by rotations and/or reflections, then only one of them is considered. As the symmetry group of a square, $D_{4}$, has order 8 (four axes of reflection and rotations at every $90^{\circ}$ ) and the symmetry group of a rectangle, $D_{2}$, has order 4 (two axes of reflection, rotations at every $180^{\circ}$ ), these orders give the maximum factors by which the number of cases can be reduced. The exact number of remaining cases can be calculated using Burnside's lemma.

Theorem 1. Let $G$ be a group acting on a set X. For $g \in G$ let $\psi(g)$ denote the number of elements of $X$ fixed by $g$. Then the number of orbits of $G$ is $(1 /|G|) \sum_{g \in G} \psi(g)$.

Here $X$ is the set of all possible choices of $n$ full tiles, and $G$ is one of the two aforementioned symmetry groups. Burnside's lemma plays a central role in counting and its proof can be found in most books on group theory and combinatorics.

In the numbering of the combinations we differ slightly from the approach in [2] and [19]. The combinations are represented as binary strings which are ranked as described in Section 3. In the next step of the proof algorithm the combinations are considered in the order of their ranks, and combinations that have a symmetric combination with a smaller rank are skipped. Contrary to the approach in [2] and [19], all combinations are ranked, not only those that are not skipped. There are two reasons for this. First, given a rank of a combination, we can immediately construct the combination without going through all combinations with smaller ranks. Second, in the next steps we do not have to start from the first combination; this is especially important if there is a huge number of combinations and the computations are distributed over several computers.

\subsection{Eliminating Combinations}

Next we try to eliminate combinations that cannot contain a packing with minimum distance at least $d_{\text {low }}$. The value of $d_{\text {low }}$ used in the computations must be sufficiently smaller than $d_{n}$ so that the elimination process works correctly, see below. We carry out the elimination by reducing parts of full tiles. The area of a full tile that has not yet been reduced is called active. Initially the whole area of full tiles of a combination is active. Throughout the paper, active areas are closed areas. The active area is reduced by considering the active areas of other full tiles. Since only the active area of a full tile can contain a point in an optimal packing, a combination can be eliminated if the active area of a full tile becomes the empty set.

In [2] and [19] each full tile is quantized into rectangles horizontally and vertically; in [17] a slightly more efficient approach is described with quantization in only one direction. In this work we present an even more efficient approach, where no such subdivision takes place, but the active area of a given full tile is considered as a polygon. An algorithm that solves the following problem is now needed. (Interestingly, when we start with convex polygons-squares and rectangles - the polygons stay convex after all reductions.) 
Reducing Polygons. Given two polygons, $A$ and $B$, and a distance $d$, reduce $B$ to another polygon $B^{\prime}$ such that $B^{\prime \prime}=B \backslash B^{\prime}$ is at distance less than $d$ from all points in $A$.

Then, if $A$ and $B$ are active areas, $B$ can be reduced to $B^{\prime}$ since a point in $B \backslash B^{\prime}$ would be at a distance less than $d$ from a point in $A$. Of course, we would like the area of $B^{\prime}$ to be as small as possible, but this is no absolute requirement.

We first prove some basic results which help in developing an algorithm. First, we note that we need only consider the vertices of $A$ in the following sense.

Lemma 1. If a point $P$ is at a distance less than $d$ from the vertices of a polygon $C$, it is at a distance less than $d$ from all points in $C$.

Proof. Draw a circle of radius $d$ around $P$. All vertices of the polygon $C$ are within this circle, and hence this holds for all points in $C$.

Theorem 2. Assume that $B_{1}, B_{2}, \ldots, B_{k}$ are distinct points on the boundary of $B$, that $B_{i} B_{i+1}$ for $2 \leq i \leq k-2$ are edges of $B$, and that $B_{1} B_{2}$ and $B_{k-1} B_{k}$ lie on edges of $B$. If the points $B_{i}, 1 \leq i \leq k$, are at a distance less than $d$ from all vertices of $A$, then the points in the polygon $B^{\prime \prime}$ obtained from these vertices are at a distance less than $d$ from all points in $A$.

Proof. Take any vertex of $A$; then it follows from Lemma 1 that this vertex is at a distance less than $d$ from all points in $B^{\prime \prime}$. Now take any point in $B^{\prime \prime}$ and apply Lemma 1 again: it follows that this point is at a distance less than $d$ from all points in $A$. This completes the proof.

So, to be able to apply Theorem 2 to reduce the polygon $B$, we need to find consecutive vertices of $B$ that are at a distance less than $d$ from all vertices of $A$. If this holds for all vertices of $B$, we get $B^{\prime}=\varnothing$ (and so this particular combination is eliminated).

Furthermore, when the consecutive edges are found, we also need to look at the edges leading to the outermost vertices to see how far away $B_{1}$ and $B_{k}$ can be placed. We then need to calculate how circles with radius $d$ and centers at the vertices of $A$ cut those edges.

In implementing an algorithm for this problem, one should note that (surprisingly, maybe) the vertices of $B$ that are at a distance less than $d$ from all vertices of $A$ need not occur in one sequence. Although rare, this anomaly in fact occurred in our computations. In Fig. 1 an example is given where a point $P$ is at a distance greater than $d$ from exactly two vertices of a convex polygon, $V$ and $V^{\prime}$, and these are not adjacent. If this happens, then one of the sequences is chosen to get $B^{\prime}$.

Note that when implementing the polygon reducing algorithm we have to be careful with the finite precision of the floating point arithmetic. The following two operations that the finite precision affects occur in the programs:

1. Is the distance between two points less than $d_{n}$ ?

2. What are the intersections between a given line (in the form of two points lying on the line) and a given circle (with radius $d_{n}$ and a given center point)? 


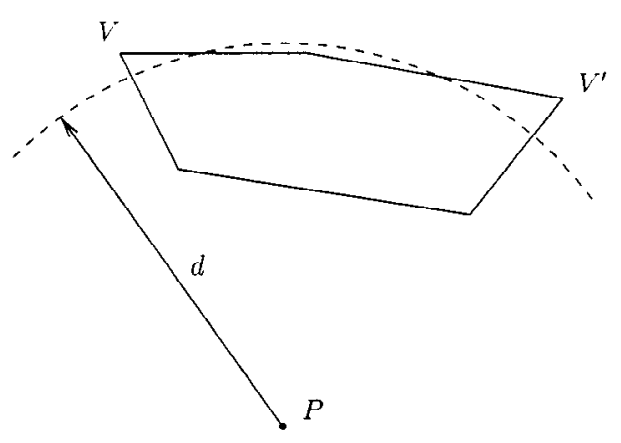

Fig. 1. A special case.

In our computations we used IEEE double-precision floating point arithmetic. The correctness of answers to the first question is taken care of by the fact that we replace $d_{n}$ by $d_{\text {low }}$ in the question. (It is important to notice that we do not require the exact answer to this question, but if we answer yes, that must be the right answer.) In all cases, we have chosen $d_{\text {low }}$ so that $d_{n}-d_{\text {low }}>10^{-9}$, which is greater than our estimated upper bound on the inaccuracy of the floating point arithmetic for this problem, $e_{1}=10^{-15}$ (which again exceeds the true inaccuracy).

The second question comes up in reducing the active area. Figure 2 describes an error that may occur if the inaccuracy of the computer arithmetic is not dealt with properly. We use the notation from Theorem 2 and the discussions around it. Again, we remember that we use $d_{\text {low }}$ instead of $d_{n}$.

Let $B_{1}$ and $B_{k}$ (everything in this discussion works similarly for $B_{k}$ ) be the points between which an edge of the reduced polygon should be inserted if we were to use $d_{n}$ in the reduction algorithm. However, as we use $d_{\text {low }}$, we would with exact precision get the vertex $B_{1}^{\prime}$. In Fig. 2 we have drawn an error circle around $B_{1}^{\prime}$ to show the error area in

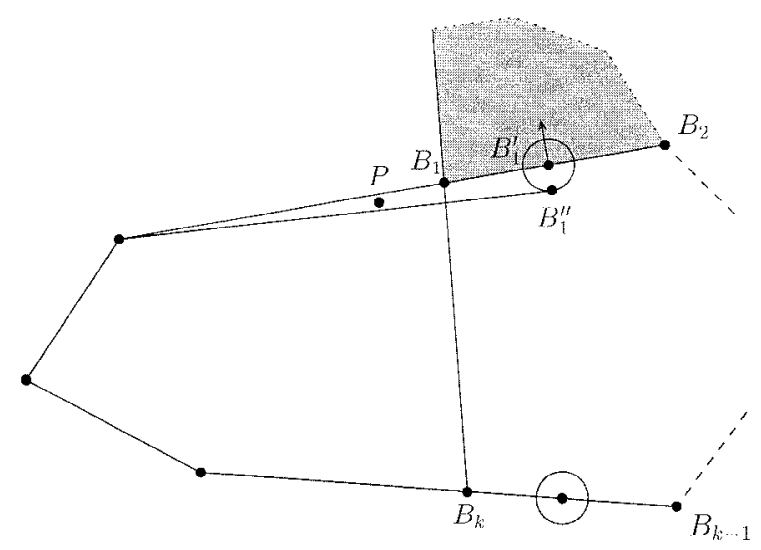

Fig. 2. Correction needed in the polygon cutting algorithm. 

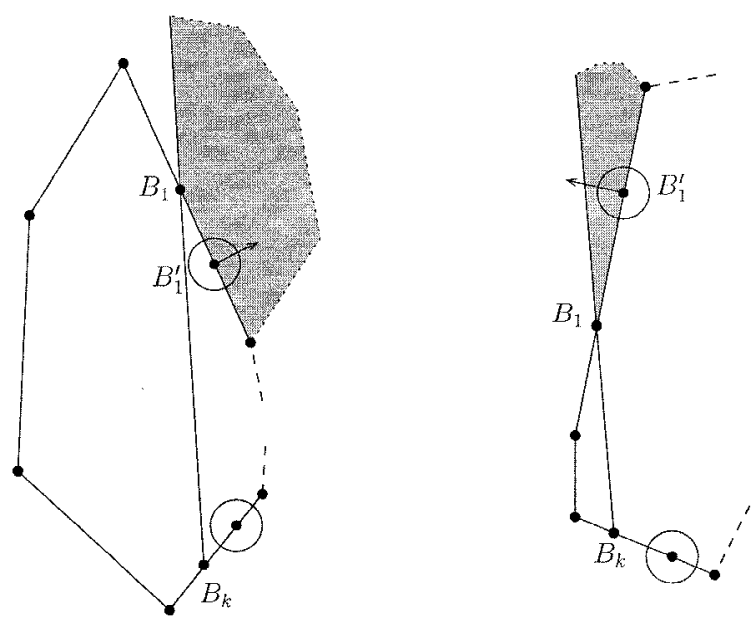

Fig. 3. Different situations for correction.

which the point as calculated by the computer will reside. Then the new vertex might be $B_{1}^{\prime \prime}$ with, for example, $P$ incorrectly outside the new active area. Therefore, a correction is necessary.

Calculations of the radius of the error area gave a rough upper bound of $e_{2}=10^{-13}$. Hence $d_{n}-d_{\text {low }}>e_{2}$ (see above) and $B_{1}$ is outside the error area. It is then enough to make a correction in the direction perpendicular to the edge through $B_{1}$ and $B_{1}^{\prime}$ by an amount greater than $e_{2}$. We used a correction by $10^{-10}$. The shaded area in Fig. 2 shows where the new vertex should be placed for correctness. Depending on the directions of the vertices we get somewhat different situations; the two extremes are depicted in Fig. 3. Note that in the second of these, we end up outside the permissible area if too large a correction is made. In that case, it is not difficult to show that the points on the line through $B_{1}$ and $B_{k}$ and on the boundary of the permissible area are at a distance greater than $d_{n}$ from some point in the active area which is used to get the reduction. Then, since all points in the same active area are at a distance less than or equal to $d_{\text {low }}$ from $B_{1}^{\prime}$, it follows that after a correction of length less than $d_{n}-d_{\text {low }}$ we are still in the permissible area (this holds, since $e_{2}<d_{n}-d_{\text {low }}$ ).

Note that we get some new active area because of the correction carried out; the important thing is that we never reduce an area that should not be reduced. The infinitesimal extra area that is added due to the correction has no noticeable influence on the overall performance of the algorithm.

In the reduction process each full tile is considered in turn to reduce the active area of other full tiles. This process can be accelerated by ruling out pairs of tiles with an active area that are so far from each other that no reduction can ever take place. After each tile has been considered, we repeat the whole procedure until an active area becomes empty and the combination is eliminated or the number of rounds reaches a given number (we used 20 in most cases). If the maximum number of rounds was achieved, then there is either an optimal packing with points in the remaining active areas of the combination, 
or we need some more sophisticated tools to reject the combination. We now discuss such advanced methods.

\subsection{Eliminating Hard Cases}

The previous step may fail to eliminate a combination that cannot contain an optimal packing especially when there are several good near-optimal packings close to each other. We can then simply continue the reduction process for some more rounds but this very seldom leads to the desired result.

A better approach — following the ideas in [2] and [19]—is to split the problem into subcases and apply the polygon reduction method to each subcase. This is done by choosing a full tile and dividing this into several rectangles, which are considered one by one (as initially containing all the active areas of that full tile). If all cases are not eliminated, we can do the same procedure for another full file, but now we can initially remove the rectangles that were eliminated in the previous rounds. This is exemplified in Fig. 4. This procedure was automatized, mostly using a division of tiles into 15 rectangles. In this way we were able to eliminate all combinations that do not hold an optimal packing.

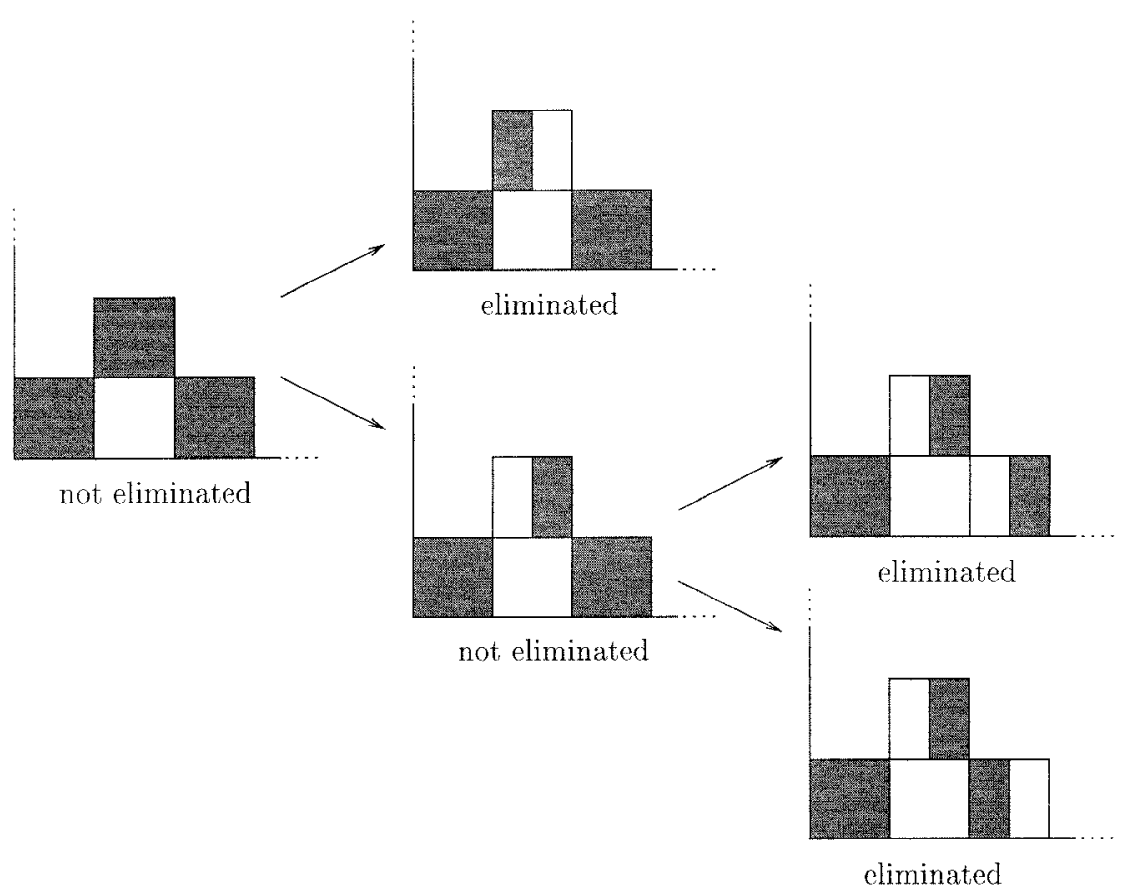

Fig. 4. Dividing the elimination problem into subcases. 


\subsection{Proving Optimality and Uniqueness}

In the final step of the optimality proof we use the method developed in [17]; see that paper for the proof of its correctness. It is based on a method used by Schaer to prove (manually) optimality of the densest packing of nine circles [21]; later Kirchner and Wengerodt [10], [28]-[30] and de Groot et al. [2], [19] used similar methods (the latter in computer-aided proofs).

At this stage of the proof algorithm-having reduced most of the active area in the combinations that we were not able to eliminate in the previous steps-we know the (small) regions in which points of an optimal packing can reside. For a given combination, we take the best known packing with the points within the remaining active areas, and try to prove that there are no better such packings.

The task of showing that such a packing with the points in the active areas indeed exists is in itself a nontrivial task. To do this, we guess (this is often obvious) which points are at minimum distance from each other and construct the corresponding system of equations. In this system there is one equation for each pair of points conjecturally at the minimum distance from each other (stating that their distance from each other equals the minimum distance). The variables of the system are the minimum distance and the coordinates of the points. Each point lying on the edge of the square has only one variable, and points lying in the corners of the square have no variables. If the guessed packing has symmetries, these provide additional equations.

In many cases (especially when there are strong symmetries in the solution), the system can be solved symbolically. The exact values of $d_{n}$ of small optimal packings are given in Table 2.1 of [13] for $n \leq 20$ and in Table 2 for $d_{23}, d_{24}, d_{25}$, and $d_{27}$ (clearly, until we are finished with the results in this paper, we only know that these are minimum distances corresponding to best known packings). The existence of such packings within the remaining active areas can be verified easily, because the coordinates of the points can be computed symbolically. In the cases where the exact values cannot be solved, we solve the system numerically to get approximate positions of the points of a solution, $\mathbf{p}_{i}^{\prime}$. In these cases, using the interval Newton/bisection program INTBIS [9], we prove that such a packing really exists with points $\mathbf{p}_{i}$ for which $\left|\mathbf{p}_{i}^{\prime}-\mathbf{p}_{i}\right|<d^{\prime}$ holds for some small positive $d^{\prime}$. We used $d^{\prime}=10^{-10}$.

Table 2. Exact and approximate values of $d_{n}$, $21 \leq n \leq 27$.

$$
\begin{aligned}
& d_{21} \approx 0.2718122553 \ldots \\
& d_{22} \approx 0.2679584015 \ldots \\
& d_{23}=\frac{\sqrt{6}-\sqrt{2}}{4 \sqrt{2}} \\
& d_{24}=\frac{\sqrt{2}+\sqrt{2}}{1+2 \sqrt{3}} \\
& d_{25}=\frac{1}{4} \\
& d_{26} \approx 0.2387347572 \ldots \\
& d_{27}=\frac{\sqrt{89}}{40}
\end{aligned}
$$


In some cases the system of equations is overdetermined and INTBIS cannot be used directly. In such a case we can try to use geometrical techniques to reduce the system into a nonoverdetermined system of equations suitable for INTBIS. For the best known packing of 26 circles, which will turn out to be optimal, we reduced the system into a single equation in one variable.

Next we draw congruent error squares (circles are used in [2] and [19], but squares were indeed used by Schaer and others in [10], [21], and [28]-[30]) of side $r$ around the points $\mathbf{p}_{i}^{\prime}$ (which are in the centers of the error squares), such that the active area remaining from the previous steps of the algorithm is within these squares (this requirement must also hold if the squares are drawn around the points $\mathbf{p}_{i}$ ). For a point on a side, only a half square is used, and for a point in a corner, a quarter of a square is used. All squares have the same size and should preferably be as small as possible. It is important that a square that is around a point in the interior of the unit square fits completely within the unit square (both with $\mathbf{p}_{i}^{\prime}$ and $\mathbf{p}_{i}$ as a center).

Finally, an elimination procedure resembling that used earlier to eliminate combinations is started. We used the elimination procedure described and proved in [17], where each active area is quantized into rectangles in one direction. (It should be possible to use the polygon reduction algorithm presented here, but the correction step described in Fig. 2 may introduce some difficulty.) The elimination procedure from [17] can be used to prove optimality by induction whenever the following theorem can be applied. The maximal error in calculations is denoted by $e$. The value of $e$ is comparable with $e_{1}$ and $e_{2}$ discussed in Section 2.3. See [17] for a proof of the theorem.

Theorem 3. Assume that $d_{\text {low }}+2 d^{\prime}+e<d_{n}$. If the active area around the points $\mathbf{p}_{i}^{\prime}$ can be reduced by the elimination process (with lower bound $d_{\text {low }}$ ) to fit within squares of side qr, with $0<q<1$ (with the points $\mathbf{p}_{i}^{\prime}$ in the centers), then the guessed packing with the points $\mathbf{p}_{i}$ is the best packing within the original error squares. Furthermore, there are no other equally good such packings.

It should be emphasized that the theorem can only guarantee a packing to be best among packings with the points within the original error squares, that is, to be locally optimal. If this procedure is applied to several remaining combinations, the best solutions found have to be compared to find the global optimum (optima).

\section{The Results}

This section contains the results of our computer runs. We both verify the results in [2] and [19] for $n \leq 20$ and consider cases up to $n=27$. We also provide new proofs for the cases $6 \leq n \leq 9$ (the case $n=7$ was considered in [17]). Some data from the computer runs can be found in Table 3, in the Appendix, and electronically in the World Wide Web at 〈URL:http://www.tcs.hut.fi/pub/packings/square $\rangle$.

In Table $3, n$ denotes the number of points and $d_{\text {low }}$ denotes the lower bound on $d_{n}$ used. The tiling is also given (see Table 1). The total number of combinations considered and the number of combinations remaining after the initial elimination procedure (dis- 
Table 3. Data from the computer runs.

\begin{tabular}{rcrrrl}
\hline$n$ & $d_{\text {low }}$ & Tiling & $T$ & $T^{\prime}$ & \multicolumn{1}{l}{ Optimal combinations } \\
\hline 6 & 0.6009252 & $3 \times 3$ & 16 & 6 & See Appendix \\
7 & 0.5358983 & $3 \times 3$ & 8 & 2 & 9111110101 \\
8 & 0.5176380 & $3 \times 3$ & 3 & 1 & 4111101111 \\
9 & 0.4999999 & $3 \times 3$ & 1 & 1 & 011111111 \\
10 & 0.4212795 & $4 \times 4$ & 1051 & 1 & 43441011110100101101 \\
11 & 0.3982073 & $4 \times 4$ & 567 & 1 & 19671101101101101101 \\
12 & 0.3887301 & $4 \times 4$ & 252 & 1 & 7181011110110111101 \\
13 & 0.3660960 & $4 \times 4$ & 77 & 2 & 1531111110110111101 \\
14 & 0.3489152 & $4 \times 5$ & 9808 & 8 & 1891311110111100001111101 \\
15 & 0.3410813 & $4 \times 5$ & 3912 & 1 & 1305311111101001011011111 \\
16 & 0.3333333 & $4 \times 5$ & 1253 & 1 & 43501111111000011111111 \\
17 & 0.3061539 & $5 \times 5$ & 136080 & 7 & $7193211111110101010101010111011(2$ packings) \\
18 & 0.3004626 & $5 \times 5$ & 60645 & 2 & 2866551101110101110111010111011 \\
19 & 0.2895419 & $5 \times 5$ & 22475 & 14 & 704791110110111111001011111101 \\
20 & 0.2866116 & $5 \times 5$ & 6814 & 2 & 173561110110111111011011111101 \\
21 & 0.2718122 & $5 \times 6$ & 3578729 & 60 & 7132215111111101000101110101011111101 \\
& & & & & 7153939111111011101000101011011111101 \\
22 & 0.2679584 & $5 \times 6$ & 1465113 & 8 & 2607260111111110100010111011011111101 \\
23 & 0.2588190 & $6 \times 6$ & 288873270 & 34 & See Appendix \\
24 & 0.2543330 & $6 \times 6$ & 156484440 & 124 & See Appendix \\
25 & 0.2499999 & $6 \times 6$ & 75112674 & 136 & See Appendix \\
26 & 0.2387347 & $6 \times 6$ & 31784172 & 1008 & 92355986111011101101111011101101011011110101 \\
27 & 0.2358495 & $6 \times 6$ & 11772390 & 162 & See Appendix \\
\hline
\end{tabular}

cussed in Section 2.3 of the proof algorithm) are denoted by $T$ and $T^{\prime}$, respectively. The combinations remaining are listed in the Appendix. After the more advanced eliminating procedure (Section 2.4) only the optimal combinations remained.

The optimal combinations (both the rank and the binary string, when there are not too many) are given in the last column of Table 3 . In the binary strings the first digit denotes the leftmost, lowermost tile ( 1 for full tile, 0 for empty tile). The next digits represent the rest of the bottom row of the tiling and the rest of the rows are represented in the same way such that the last few digits denote the top row. There are $\left(\begin{array}{l}t \\ n\end{array}\right)$ possible such strings, where $t$ is the number of tiles in the tiling. These strings are ranked in inverse lexicographic order so that the first string is $11 \cdots 1100 \cdots 00$ and the last $00 \cdots 0011 \cdots 11$; the rank of the first string is 0 and the last string has rank $\left(\begin{array}{l}t \\ n\end{array}\right)-1$. For example, in a $3 \times 3$ tiling the combination 111101111 means a configuration where the center tile is empty and the other tiles are full. The rank of this combination is 4, because there are four other combinations (111111110, $111111101,111111011,111110111)$ before this combination. For efficient ranking and unranking algorithms, see, for example, [26].

For the cases $10 \leq n \leq 20$, the number of remaining combinations after the first elimination process (Section 2.3) is in some cases smaller than that in [2] and [19]. We have checked that all our remaining combinations are among those remaining in that study. There is a misprint in [2] and [19]: the number of initial combinations for $n=12$ is incorrect (267) since they indeed used 252 combinations in their search [20]. 
The part of the proof procedure that is most time-consuming is the initial elimination part of the proof algorithm (Section 2.3). In [2] and [19] about 3 CPU-hours of computation time was used for the most difficult cases for $n \leq 20$. The number of combinations increases rapidly for $n \geq 21$. Consequently, there is also a rapid increase in CPU-time needed. The four cases with $23 \leq n \leq 26$ required as much as several CPU-months of computation time on a Sun Sparcstation 2 workstation when using the elimination process described in [17]. Since $d_{27}$ is just slightly greater than the diameter of the tiles (see Tables 1 and 2), the elimination method in [17] cannot be used for $n=27$ without large computing resources. Even the increasing computing power in the foreseeable near future does not help much. However, using the polygon reduction algorithm described in Section 2.3, we were able to speed up the search remarkably, and the first (and most time-consuming) elimination stage for all cases $n \leq 27$ was completed in a month of CPU-time.

In some cases there are several combinations that lead to an optimal packing. For $n=6,23,24,25,27$ the optimal packings have points that lie exactly on the border between two or four tiles. Different combinations then converge to the optimal packings from different directions. For $n=21$ the direction of the tiling constitutes the difference between the first and the last two combinations (there are two of these due to a large area within which a loose circle can move). The same phenomenon does not occur for other tilings where the number of rows and columns are not equal, as the optimal packings in those cases are diagonally symmetric $(n=14,15,16)$ or nearly symmetric $(n=22)$.

The optimality proof outlined in Section 2.5 worked out well for all cases except $n=21$. We first suspected that the guessed packing is not optimal but it later turned out that the algorithm for the optimality proof discussed in Section 2.5 simply does not converge due to long chains of interactions and feedbacks between the error areas. However, starting from error squares of side 0.002 , the following results are sufficient to get an optimality proof by induction (a complete proof is longish and therefore left out; it follows the approach in [17]). Consider the point corresponding to the circle very close to the border in Fig. 5. Divide the error square around that point into two rectangles: $A$ consists of $\frac{7}{16}$ of the original area and is close to the border; $B$ consists of the rest of the area (and contains the point). Now if $A$ is the only initial active area around that point, the reduction procedure eliminates one of the active areas completely and thus there cannot be a solution with a point in $A$. On the other hand if $B$ is the only active area, the convergence required in Theorem 3 is achieved (the requirement that the active area has to fit within a square of side $q r$ still holds, so the error area is allowed to reach the edge between $A$ and $B$ after elimination).

The optimal packings for $21 \leq n \leq 27$ are depicted in Fig. 5 and the corresponding values of $d_{n}$ are given in Table 2. In a recent paper of Maranas et al. [11], it is claimed that there is a packing of 21 circles with $d \approx 0.27181675$; in view of the results of this paper, that value is erroneous.

\section{Conclusions}

It can always be argued whether results obtained by computers (and which cannot be checked by hand) should be trusted. Evidence of correctness of the results here are, 


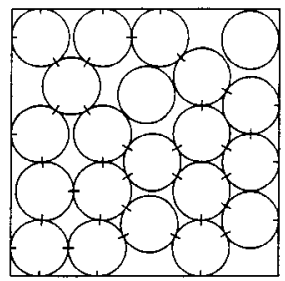

$n=21$

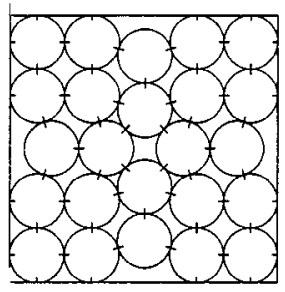

$n=24(a)$

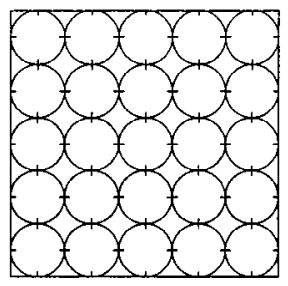

$n=25$

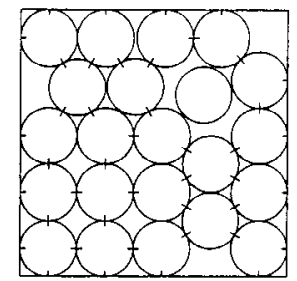

$n=22$

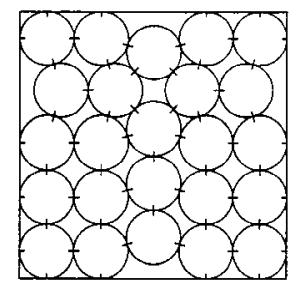

$n=24(b)$

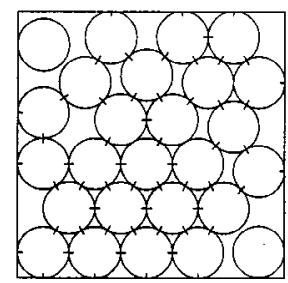

$n=26$

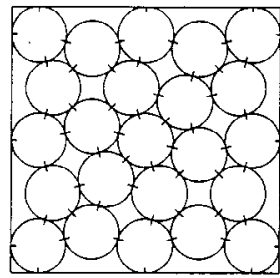

$n=23$

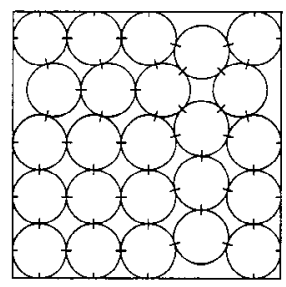

$n=24(c)$

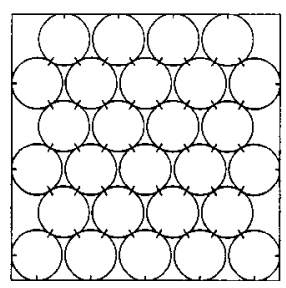

$n=27$

Fig. 5. Optimal packings for $21 \leq n \leq 27$.

however, provided by at least the following facts. First, all packings of at most 20 circles that were proved optimal in [2] and [19] turned out to be optimal in this study also. Second, all optimal packings of 21-27 circles are among those reported in [7] and [16]. Third, the square lattice packing of 25 circles has earlier been proved optimal by Wengerodt [29]. Furthermore, the manual proofs that have been published are in some cases, such as in [30], so complicated that the authors find them in practice less convincing and more error-prone than a computer-aided proof, where each step is relatively simple.

With massive computer power and further development of the algorithms, it should be possible to proceed beyond 27 circles. In at least three ways, the search gets more difficult and time-consuming when the number of circles grows:

1. The initial number of combinations grows rapidly.

2. An increasing number of combinations remaining after the initial elimination has to be considered using methods from Section 2.4.

3. A straightforward proof of optimality as described in Section 2.5 may fail and methods similar to that used for the packing of 21 circles in this paper may become necessary. 
(Note that the growth discussed is not monotonic.) For the first item, there is the following possible strategy. The square is first subdivided into two (halves) or more parts. Packings of points into these areas are then calculated and combined to reduce the number of initial combinations.

The approach in the paper can with minor modifications be used for packings in others geometrical objects. These results (primarily for packings in an equilateral triangle and a circle, see [6] and [8]) will be discussed in forthcoming papers [18].

\section{Appendix}

The ranks of the combinations remaining after the initial elimination procedure discussed in Section 2.3 are listed here. Due to the large number of combinations in the case $n=26$, these are not included; the combinations can be obtained from the authors or from the World Wide Web at 〈URL:http://www.tcs.hut.fi/pub/packings/square〉, where files with the results for all $6 \leq n \leq 27$ are available.

For $n=6$ and $n=25$ we have more than one remaining combinations with the property that all of these lead to the optimal packing. The number of optimal combinations for $n=6,23,24,25,27$ can be calculated using Burnside's lemma (Theorem 1). For example, for $n=25$ the number of combinations is the number of ways of choosing one of each of the tiles with letters A, B, C, D, E, F, G, H, and I in Fig. 6 (all other tiles are full tiles) taking symmetries into account. To avoid the abundance of optimal combinations, it would maybe have been more appropriate to choose another tiling in these cases, such as $5 \times 7$.

$\mathbf{n}=\mathbf{6}:$

Optimal combinations:

$8,11,18,23,35,41$.

\begin{tabular}{|l|l|l|l|l|l|}
\hline & & A & A & & \\
\hline & & B & B & & \\
\hline C & D & E & E & F & G \\
\hline C & D & E & E & F & G \\
\hline & & H & H & & \\
\hline & & I & I & & \\
\hline
\end{tabular}

Fig. 6. Possible optimal combinations for $n=25$. 
$\mathbf{n}=\mathbf{7}$ :

$9,13$.

$\mathbf{n}=\mathbf{8}$ :

4.

$\mathbf{n}=\mathbf{9}$ :

0 .

$\mathbf{n}=\mathbf{1 0}$ :

4344.

$\mathbf{n}=11:$

1967.

$\mathrm{n}=12:$

718 .

n = 13:

$153,397$.

n = 14:

18913, 19405, 19734, 31543, 32175, 32643, 34063, 34967.

$\mathbf{n}=15$ :

13053.

$\mathrm{n}=16$ :

4350 .

n = 17:

531789, 548782, 548793, 574897, 575732, 575742, 719321.

$\mathbf{n}=18$ :

203480, 286655.

n = 19:

35318, 35620, 37440, 68967, 69269, 70479, 70546, 70551, 70787, 71089, 93269, 95301, 150179, 151350.

n $=20$ :

$17356,45944$.

n = 21:

3929891, 6771475, 6778408, 6778425, 6779664, 6782996, 6942984, 6978773, 
7036389, 7037996, 7122790, 7132215, 7132220, 7132223, 7147036, 7153939, 7154276, 7159934, 7166315, 7169398, 7169412, 7172310, 7172536, 7206322, 8793230, 8840763, 8841405, 8998420, 8998425, 8998483, 8998500, 8998703, 8999059, 9085920, 9087527, 9098296, 9098351, 9110869, 9258071, 12370515, 12372558, 12377804, 12378895, 12577163, 12578818, 12635785, 13038882, 13101492, 13102572, 13102573, 13108095, 13110516, 13126099, 13138475, 13139799, 13210460, 13211530, 13212500, 13213545, 13219788.

$\mathbf{n}=22$ :

2607260, 3555400, 3557910, 4897651, 4899141, 5212141, 5231020, 5241328.

$\mathbf{n}=23:$

Optimal combinations:

1697836485, 1697836486, 1697836849, 1697836850, 1697849277, 1697850277, 1697850278, 1698043692, 1698043693, 1698828945, 1698828946, 1698829309, 1698829310, 1698847924, 1698848925, 1698954824, 1698986790, 1699122784, $1713852466,1713854468$.

Other combinations:

1227864664, 1227864665, 1227864672, 1227864673, 1228158594, 1690426781, 1690428330, 1690448464, 1695146683, 1695175284, 1695224203, 1695738516, $1695841476,1710688358$.

$\mathbf{n}=\mathbf{2 4 :}$

Optimal combinations (a):

881862520, 881862521, 881862528, 881862529, 881868594, 881868601, 881868602, 881877103, 881877104, 881892016, 882183430, 882183431, 882183438, 882183439, 882195859, 882195860, 882195867, 882195868, 882214357, 882214358, 882214365, 882214366, 882239097, 882239098, 882239105, 882679031, 882679038, 882679039, 882688912, 882688919, 882707410, 882707417, 882707418, 882728517, 883175899, $887507595,887507602,887526100,888019145$.

Optimal combinations (b):

878173088, 878173089, 878173096, 878173097, 878299058, 878299059, 878299066, 878299067.

Optimal combinations (c):

880216063.

Other combinations:

871779778, 871779779, 871779786, 871779787, 871905748, 871905749, 871905756, 871905757, 877220329, 877220330, 877220693, 877220694, 877226764, 877226765, 877227765, 877227766, 877235112, 877235113, 877236113, 877236114, 877250607, 877250608, 877252609, 877252610, 877259089, 877259090, 877259453, 877259454, 877265524, 877265525, 877266525, 877266526, 877273872, 877273873, 877274873, 
877274874, 877289367, 877289368, 877291369, 877415269, 877415270, 877415633, 877415634, 877428060, 877428061, 877429061, 877429062, 877446404, 877446405, 877447405, 877447406, 877471727, 877473729, 877492789, 877492790, 877493153, 877493154, 877505580, 877505581, 877506581, 877523924, 877523925, 877524925, 877524926, 877549247, 877551249, 877733220, 877733584, 877761807, 877762808, 877810740, 877811104, 877839327, 878091190, 878091554, 878217160.

$\mathbf{n}=\mathbf{2 5}$ :

Optimal combinations:

397525108, 397525109, 397525116, 397525117, 397525199, 397525200, 397525207, 397525208, 397527039, 397527046, 397527047, 397527402, 397527403, 397527410, 397527411, 397530040, 397530041, 397530396, 397530397, 397530404, 397530405, 397536322, 397537314, 397537315, 397537322, 397537323, 397580342, 397580343, 397580350, 397580351, 397586416, 397586423, 397586424, 397594925, 397594926, 397696076, 397696077, 397696084, 397696085, 397696167, 397696168, 397696175, 397696176, 397700966, 397700967, 397700974, 397700975, 397701330, 397701331, 397701338, 397701339, 397709468, 397709469, 397709476, 397709477, 397709832, 397709833, 397709840, 397709841, 397722105, 397722106, 397722113, 397723106, 397723107, 397723114, 397736522, 397736523, 397736530, 397736531, 397748951, 397748952, 397748959, 397767449, 397767450, 397767457, 397767458, 397792189, 397792190, 397792197, 397814042, 397814043, 397814050, 397814051, 397826471, 397826472, 397826479, 397844969, 397844970, 397844977, 397844978, 397869709, 397869717, 398015287, 398015294, 398015295, 398015378, 398015385, 398015386, 398018917, 398018924, 398019281, 398019288, 398027419, 398027426, 398027427, 398027783, 398027790, 398027791, 398037515, 398038516, 398054473, 398054480, 398054481, 398064354, 398064361, 398082852, 398082859, 398103959, 398131993, 398132000, 398132001, 398141874, 398141881, 398160372, 398160379, 398334945, 400825517, 400825524, 400825881, 400825888, 400834026, 400834383, 400834390, 400951029, 400969527, 401151969.

$\mathbf{n}=\mathbf{2 7}$ :

Optimal combinations:

$32827518,32827519,32827882,32827883$.

Other combinations:

11783292, 14671315, 19701872, 20569553, 21804726, 21824433, 29939496, 30198184, 30198308, 30198314, 30235506, 30401840, 30428174, 30428537, 30428538, 30431597, 32419659, 32801185, 32826168, 32827473, 32827525, 32827759, 32827837, 32827888, 32827889, 32830942, 37858000, 37858005, 37858076, 37858718, 37859293, 37859360, 37861136, 37873580, 37873586, 37892923, 37892989, 38006988, 38031367, 38038236, 38043318, 38043322, 38043327, 38043328, 38053740, 38054381, 38725757, 38726399, 38726639, 38726763, 38726769, 38729253, 38729758, 38729822, 38900428, 38902239, 38902786, 38903499, 38903862, 38903863, 38903869, 38906858, 38906903, 38906922, 38909183, 38909188, 38911003, 38911008, 38911009, 38912364, 
38913930, 39034219, 39266825, 39271567, 39526894, 39561298, 39561299, 39561662, 39561663, 39564358, 39564722, 39817431, 39817555, 39853312, 39892044, 39892168, 39910367, 39960930, 39978985, 39980273, 39980637, 39980700, 39980824, 39980860, 40322546, 40467208, 51742232, 51742874, 51743237, 51743516, 51745298, 51746297, 51761437, 51775338, 51777079, 51864810, 51891144, 51896599, 51907129, 51910101, 51915523, 51916461, 51919332, 51920337, 51923397, 51925654, 51925663, 51927474, 51927478, 51927483, 51927769, 51927908, 51927919, 51927947, 51928839, 51930249, 51940744, 51940751, 51940810, 51940817, 51942987, 51943381, 51943416, 51943417, 51943423, 51943588, 51943601, 51944746, 51944966, 52609913, 52752840, 52757321, 52770186, 52786395, 52797910, 53410640, 53444104, 53701587, 53701985, 53703778, 53704575, 53704581, 53704751, 53704759, 53705036, 54178411, 84270113, 87655593.

We finally give one optimal combination for each of the cases not listed in Table 3:

$\begin{array}{lll}\mathbf{n}=\mathbf{6}: & 8 & 111101010 \\ \mathbf{n}=\mathbf{2 3}: & 1697836485 & 111011101011111001000010110101111011 \\ \mathbf{n}=\mathbf{2 4}(\mathbf{a}): & 881862520 & 111011111011110011000000111011111011 \\ \mathbf{n}=\mathbf{2 4}(\mathbf{b}): & 878173088 & 111011111011000000111011110011111011 \\ \mathbf{n}=\mathbf{2 4}(\mathbf{c}): & 880216063 & 111011111011000000111011101011111011 \\ \mathbf{n}=\mathbf{2 5}: & 397525108 & 111011111011111011000000111011111011 \\ \mathbf{n}=\mathbf{2 7}: & 32827518 & 111011101101111011101101111011101101\end{array}$

\section{References}

1. H. T. Croft, K. J. Falconer, and R. K. Guy, Unsolved Problems in Geometry, Springer-Verlag, New York, 1991.

2. C. de Groot, M. Monagan, R. Peikert, and D. Würtz, Packing circles in a square: a review and new results, in P. Kall (ed.), System Modelling and Optimization (Proc. 15th IFIP Conf., Zürich, 1991), pp. 45-54, Lecture Notes in Control and Information Sciences, Vol. 180, Springer-Verlag, Berlin, 1992.

3. C. de Groot, R. Peikert, and D. Würtz, The Optimal Packing of Ten Equal Circles in a Square, IPS Research Report 90-12, ETH, Zürich, 1990.

4. L. Fejes Tóth, Lagerungen in der Ebene, auf der Kugel und im Raum, 2nd edn., Springer-Verlag, Berlin, 1972.

5. M. Goldberg, The packing of equal circles in a square, Math. Mag. 43 (1970), 24-30.

6. R. L. Graham and B. D. Lubachevsky, Dense packings of equal disks in an equilateral triangle: from 22 to 34 and beyond, Electron. J. Combin. 2 (1995), A1, 39pp.

7. R. L. Graham and B. D. Lubachevsky, Repeated patterns of dense packings of equal disks in a square, Electron. J. Combin. 3 (1996), R16, 17 pp.

8. R. L. Graham, B. D. Lubachevsky, K. J. Nurmela, and P. R. J. Östergård, Dense packings of congruent circles in a circle, Discrete Math. 181 (1998), 139-154.

9. R. B. Kearfott and M. Novoa, III, Algorithm 681: INTBIS, a portable interval Newton/bisection package, ACM Trans. Math. Software 16 (1990), 152-157.

10. K. Kirchner and G. Wengerodt, Die dichteste Packung von 36 Kreisen in einem Quadrat, Beiträge Algebra Geom. 25 (1987), 147-159.

11. C. D. Maranas, C. A. Floudas, and P. M. Pardalos, New results in the packing of equal circles in a square, Discrete Math. 142 (1995), 287-293.

12. H. Melissen, Densest packing of six equal circles in a square, Elem. Math. 49 (1994), 27-31. 
13. H. Melissen, Packing and Covering with Circles, Ph.D. thesis, University of Utrecht, 1997.

14. M. Mollard and C. Payan, Some progress in the packing of equal circles in a square, Discrete Math. 84 (1990), 303-307.

15. L. Moser, Problem 24 (corrected), Canad. Math. Bull. 3 (1960), 78.

16. K. J. Nurmela and P. R. J. Östergård, Packing up to 50 equal circles in a square, Discrete Comput. Geom. 18 (1997), 111-120.

17. K. J. Nurmela and P. R. J. Östergård, Optimal packings of equal circles in a square, Proceedings of the Eighth International Conference on Graph Theory, Combinatorics, Algorithms, and Applications, in press.

18. K. J. Nurmela and P. R. J. Östergård, Packing congruent circles in an equilateral triangle, in preparation.

19. R. Peikert, Dichteste Packungen von gleichen Kreisen in einem Quadrat, Elem. Math. 49 (1994), 16-26.

20. R. Peikert, private communication.

21. J. Schaer, The densest packing of nine circles in a square, Canad. Math. Bull. 8 (1965), 273-277.

22. J. Schaer, On the packing of ten equal circles in a square, Math. Mag. 44 (1971), 139-140.

23. J. Schaer and A. Meir, On a geometric extremum problem, Canad. Math. Bull. 8 (1965), 21-27.

24. K. Schlüter, Kreispackung in Quadraten, Elem. Math. 34 (1979), 12-14.

25. B. L. Schwartz, Separating points in a square, J. Recreational Math. 3 (1979), 195-204.

26. D. W. Stanton and D. E. White, Constructive Combinatorics, Springer-Verlag, New York, 1986.

27. G. Valette, A better packing of ten circles in a square, Discrete Math. 76 (1989), 57-59.

28. G. Wengerodt, Die dichteste Packung von 16 Kreisen in einem Quadrat, Beiträge Algebra Geom. 16 (1983), 173-190.

29. G. Wengerodt, Die dichteste Packung von 25 Kreisen in einem Quadrat, Ann. Univ. Sci. Budapest. Eötvös Sect. Math. 30 (1987), 3-15.

30. G. Wengerodt, Die dichteste Packung von 14 Kreisen in einem Quadrat, Beiträge Algebra Geom. 25 (1987), 25-46.

Received February 11, 1998, and in revised form December 17, 1998. 